\title{
The Design and Implementation based on IOS Pocket Closet
}

\author{
Zheng Ruiying
}

Institute of mathematics and computer science, Jiangxi Science and Technology Normal University

Key words: Pocket closet; Objective - C; IOS; SQL Server; PHP

Abstract: Pocket closet is mobile application software developed based on Objective - C language, which can help users manage a wardrobe. When users don't know how to match clothes, the software recommends collocation through user testing results; Users don't have to dig out tie-in dress, but through the software to match clothes. It is convenient with simple operation. Users can also shop clothes, and buy their clothes online, at the same time users can discuss topics of common interest with other users. Pocket closet is a really convenient mobile application.

\section{THE INTRODUCTION}

People all have the love of beauty in their hearts. In everyone's closet, there are many clothes forgotten in a corner only through one or two times out. Later, suddenly found them in the wardrobe. Many people have such trouble: there are so many clothes in the closet, but don't know how to match. Sometimes you want to buy clothes, but just don't know how to go out to stroll. Based on these problems, I would like to develop this software which can solve the above problems.

Pocket closet is a software closely related to life, easy to operate, and can help users to manage wardrobe. With the development of science and technology and network, input information clothes is simple, and the user can manually put phone clothes photos uploaded to the application or use of uploaded to the application by mobile phone camera for user-friendly management wardrobe and match clothes later. Based on the common trouble mentioned, in order to solve these problems, I developed the application.

Pocket closet application uses Objective - C language to develop the program, use UIKit framework to build all pocket closet framework, mysql database to build the background to storage data required for the application. As long as the user mobile phone is equipped with the software, users can tie-in dress, anytime and anywhere, see clothes, buy clothes online, and let user be comfortable and save worry to follow fashion trends.

\section{THE APPLICATION ARCHITECTURE}

Pocket closet is based on IOS application. It uses Objective - C language, develop in Xcode 5.0.2 development environment for ordinary users to match dress fashion management and purchasing.

\section{A. Application run time environment}

Operating system: OS X 10.8.5

Database: the MYSQL 5.0

Development environment: Xcode 5.0.2

Server: MAMP2.2

\section{B. Application development technologies}

1. IOS profile

IOS is the world's most advanced mobile operating system, which constantly redefines what people can use mobile devices to do. In short, iOS SDK and Xcode IDE are easy to allow developers to create revolutionary mobile applications.

2. UIKit framework structure 
UIKit framework provides the iOS required classes to build and manage application's user interface. It provides an application object, event processing, imaging model, window, view and controller and is designed for touch screen interface.

MVC design pattern is a very classic design pattern. Through MVC design pattern, application logic can be divided into data model, controller logic, and view. Model data model is mainly the logic for application's data and operation data. View is model data displayed on interface application, which allows the user to edit to it. Controller object is coordinator between application view object and object model, which has the effect of the bridge to show UI logic control with data interaction.

3. Objective - C

Objective - $\mathrm{C}$ is an object-oriented editor language, which is written with iOS program, but it is the language of derived by $\mathrm{C}$ language, which not only inherits the characteristics of $\mathrm{C}$ language, but also expands C language. This is a superset of $\mathrm{C}$ programming language, and provides object-oriented functions and dynamic operation. Objective - $\mathrm{C}$ inherits syntax, basic types and flow $\mathrm{C}$ control statements, and increases syntax to define classes and methods. It also adds to the management of graphics object and objects constants, and at the same time provides dynamic typing and dynamic binding, which delays the many responsibilities until run time language level support.

\section{MYSQL database}

In the application, we only need to store clothes information. With relevant information, user information posts topics, and comments on the topic. MYSQL database just conforms to this characteristic, so I chose it in application as application database.

\section{APPLICATION NEEDS ANALYSIS}

According to the using characteristics of wardrobe, the application can be divided into the following several modules: walk module, collocation module, share communication module, recommend module. Application of function module diagram is shown in Figure 1.

Walk function module mainly has a list of clothes list, clothes introduction and clothes details. Walk function module diagram is shown in Figure 2.
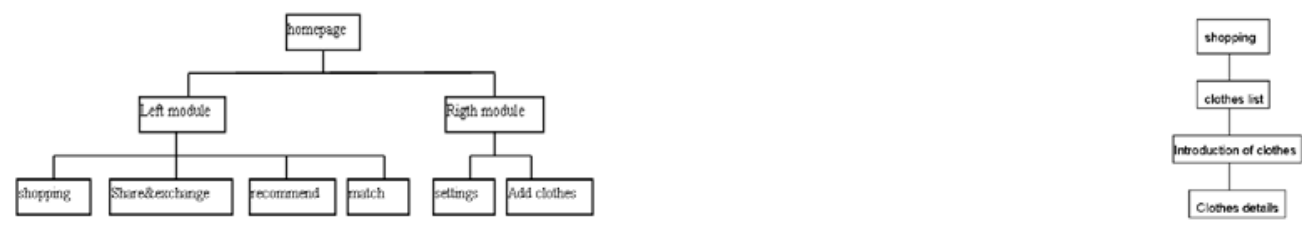

Figure 1 Application function module diagram Figure 2 Walk function module diagram

Share communication function module mainly includes log in, registration, issue list, check topics, reviews topics, post topics. Share communication function module diagram is shown in Figure 3.

Collocation function module is mainly tie-in, custom tie-in, choose clothes, show collocation, and choose a topic. Collocation function module diagram is shown in Figure 4.
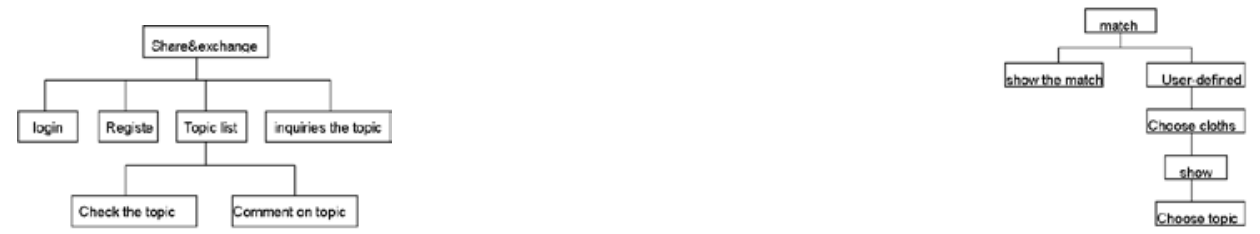

Figure 3 Share communication function module diagram Figure 4 collocation function module chart 


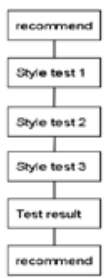

\section{Figure 5 Recommend function module diagram}

Recommend function module mainly includes testing style one, style test two, style test three, test results, recommendation. Recommend function module diagram is shown in Figure 5.

\section{APPLICATION IMPLEMENTATION}

\section{A. Walk module}

Walk function module is mainly used for online clothes purchase, and achieves to buy clothes by mobile phone which is convenient and saves time.

The difficulty in this function module is json parsing and image loading problem. For json parsing the difficulty, I make a request through the network, then with JSONObjectWithData:(NSData *)data options:(NSJSONReadingOptions)opt error:(NSError **)error method to parses the data, and then use NSDictionary dictionary to store data. Because we can load more pictures with up and down refreshment, it will bring a problem. If more pictures, loading speed is slow, and pictures are showed slow, and the sense that gives a user is very slow with quiz and the user experience is not good. In order to solve this problem, I introduce UIImageView + WebCache file. As long as we import the file header files in the program, and then display images by using setImageWithURL method, loading problem is solved.

\section{B. Collocation module}

Collocation function mainly is the realization of user's own clothes match operation. Users don't have to dig out the collocation of clothes. As long as choose clothes via mobile phone, check collocation, then check the match result, if he does not like it, match again.

Collocation design is that if the user is the first time into collocation function module, only theme words are on the first interface, and there is no corresponding collocation pictures each topic. Users click the button on the upper right corner, enter a custom interface, and the interface is designed to connect to the database, which will input information table in database information to download and store down. According to type attribute classification, divide display images information into five classifications: clothes, beauty makeup, accessories, shoes, bags. The user can select images in each category to match, and the same images can't be chosen twice, and you can delete selected images. After selection, click the button in the top right corner, enter the collocation browsing interface, and if the user is satisfied with the collocation, which can click the button on the upper right corner, enter the theme selection. The user matches clothes in topic selection, classify them. If the suit is suitable for wear to work or off work. The user also can input topic separately. Good choice and then click the button below enter the first interface, and users can see their collocation clothes pictures at this moment, choose the theme selected, and then tie-in images will be shown just below. In this function module, the user can match their clothes, so you don't have to dig out the collocation of clothes, and the user can also match clothes anytime and anywhere.

\section{Match clothes function module}

User's choice of clothes source is for the user to add clothes. Clothes interface contains a selection of five categories: clothing, beauty makeup, shoes, bags and accessories. The user can freely choose 
the items to complete the match, and can delete the selected item. The program will determine the same items cannot be selected twice. After selection, click the next step on the upper right corner, jump to display collocation, and the user interface mainly displays good effect pictures.

In this function module, in order to allow users to let users more convenient and comfortable, according to classification, we can control the classification of the slide to reveal hidden. The effect of the implementation of the method is implemented on UIScrollerView by adding UILabel. Controls stack is to achieve good effect view. UIScrollerView is mainly to realize the effect of sliding. UILabel mainly shows classification. Classification corresponding picture is also the difficulty and key. Connect to the database, load and store the input the data in information clothes table. According to the type attribute, classify the pictures, which is to realize the key point. After classification, the pictures are classified; In the process of development, how to realize the corresponding image display is also a difficult point, and the solution to this problem is how many kinds of statistical classifying. On the basis of the original view, create child views after statistical classification number and use classification and storage of data to display the data corresponding on each child view. Of course, these data show carrier is UIButton so as to trigger the click event, then click on the event, I realized that immediately after clicking, the selected images shows below, so the layout is very important in this function module.

The main implementation code:

/ / delete button respond events

- (void)removeit:(UIButton *)sender\{

count $=$ count -1 ;

UIButton *butts = nil;

for(UIButton *btn in self.btnArr)\{

if(btn.tag $==$ sender.tag) \{

[bth removeFromSuperview];

butts = btn;

\}

\}

[self.btnArr removeObject:butts ];//delete images from the array

UIImageView * tempImageView = nil;

For (UIImageView * image in the self. ImageArr) \{

If (image. The tag = = sender. Tag) \{

[image removeFromSuperview];

[self. Clothess removeObject: image. Image]; / / delete pictures

TempImageView = image;

\}

\}

[the self imageArr removeObject: tempImageView]; / / delete pictures from pictures in the array

// used to implement the slide to delete, is suitable for automatically move to the left

For (UIView * obj in [the self scrolingdelega subviews]) \{

[obj removeFromSuperview];

\}

/ / used to reload the view

For (int $I=0 ; I<$ self. ImageArr. Count; $i++)\{$

UIImageView $*$ tempImage = self. ImageArr [I];

TempImage. Frame = CGRectMake $(5+I * 72,10,72,67)$;

[the self scrolingdelega addSubview: self imageArr [I]].

\}

For (int $I=0 ; I<$ self. BtnArr. Count; $i++$ ) \{ 
UIButton * butts = self. BtnArr [I];

Butts. Frame = CGRectMake $(3+72 * I, 6,15,15)$;

[the self scrolingdelega addSubview: self btnArr [I]].

\}

\}

// click next respond to events

- (void) finishOK \{

If ([self scrolingdelega subviews]. Count $==0)\{$

UIAlertView * alert = [[UIAlertView alloc] initWithTitle: nil message: @ "please add clothes!" the delegate: self cancelButtonTitle: nil otherButtonTitles: @ "good," nil];

[alert show];

[alert release]; / / hint didn't add clothes

\} else \{

Init MatchfinishViewController * finishViewControll = [[MatchfinishViewController alloc]].

[the self navigationController pushViewController: finishViewControll animated: YES]; / / jump interface

\}

\}

2. Choose topic and display module

After collocation, get into choose topic interface. The user can choose subject or input subject. Display collocation interface after chosen the topic. The interface contains subject classification. If the user has added the collocation of the classification, show collocation figure under the corresponding classification, and the user can click on the image to get into the browsing.

Choosing theme function module design is that the user can select theme and can also manually enter the theme. Manually add theme will be automatically added to the list of theme, and when getting to the theme for the second time in interface, manually input it to the theme. Line up an interface to other topics when the theme list full. The user can slide to view your subject. How to achieve this is the emphasis and difficulty to choose the function module. My realization method is to add a child views on the original view, add UIScrollView in this child views to slide around to implement, add on UIButton button to UIScrollView to display the theme, adn when the number of topic is greater than 12, UIScrollView can slide and add the theme. And add the data well match with the theme to the database.

Show collocation function module design idea is to connect the database, load and save the data in match table. According to the classified storage of theme attribute in the table, match images classification. In this function module, adopt the same method as tie-in dress function module to display the theme. While sliding theme, the following load content changes, and we also can check the original match. The function modules' difficulty is how to realize theme change, and the following content changes. My method is to add UIScrollView to the bottom of the theme, determine the width of UIScrollView according to the load type theme number, and realize each topic on UIScrollView accounted for a page. When UIScrollView slides around, there is a scrollViewDidScroll method for special monitoring events, and when UIScrollView slides around, display collocation image in this way.

\section{SUMMARY}

This app has the advantage that when user is in use, the vast majority of functions can be accessed, and they don't have to worry about permissions. For the problems like forget what clothes you have, don't know what to wear clothes in the morning, don't know tie-in dress, they are resolved in the app. The app also adds the function of share exchange, so that the user can communicate with other 
users to share their tie-in tips, discuss how to match clothes, set up a bridge of communication, and also broaden the user's circle of friends. The app uses simple and practical, easy operation, intelligent and humanization. Of course, this app has shortages. In collocation module, if use $3 \mathrm{~d}$ figure to match clothes, give a person better visual effect. In the recommendation module, the user sees most words, while if there are pictures in recommend collocation, the recommended collocation effect is better.

\section{Acknowledgment}

This work is financially supported by the Social Science Planning Project of Jiangxi Province under Grant NO. 14TQ04

\section{REFERENCE}

[1] Dave Mark, Jack Nutting, Jeff LaMarche ios 5 cs (second edition) [M]. Beijing: People's Posts and Telecommunications Publishing House, 2012-12.

[2] Mark Dalrymple, Scott Knaster Objective - C based tutorial (second edition) [M]. Beijing: People's Posts and Telecommunications Publishing House, 2012-10.

[3] Teodor Danciu, Lucian Chirita. The Definitive Guide to JasperReport. The 2007-8.

[4] Liu Jieyu. A game design and implementation based on iOS [D] University of Electronic Science and Technology, 2012.

[5] Huang Xiaoguo. Testing technology in the process of software development [J] Computers 1995, 03 